\title{
A note on the diameter of convex polytope
}

\author{
Yaguang Yang*
}

November 6, 2020

\begin{abstract}
This short note extends a recent result (Bonifas et al, On sub-determinants and the diameter of polyhedra, Discrete Computational Geometry, 52, 2014) of an upper bound of the diameter of a convex polytope defined by an integer matrix to a similar upper bound of the diameter of a convex polytope defined by a real matrix. It also shows, by an example, that the new bound may be better than the ones of Bonifas et al.
\end{abstract}

Keywords: diameter of convex polytope, linear programming.

\section{Introduction}

A polytope $P=\left\{x \in \mathbb{R}^{n}: A x \leq b\right\}$ is defined by a $m \times n$ matrix $A$, a vector $b$, and $m>n$. Let $x^{*} \in P$ denote a vertex of $P$ which satisfies (a) the system of inequalities $A x^{*} \leq b$ holds and (b) $n$ equalities hold for some linear independent rows of $A$. Two vertices $x^{*}$ and $y^{*}$ are neighbours if they are connected by an edge of $P$, which is defined by $n-1$ linearly independent rows of $A$ where the equalities hold for both $x^{*}$ and $y^{*}$. In this way, any two vertices on $P$ are connected by a path composed of a series of edges. The diameter of $P$ is the integer that is the smallest number of edges between any two vertices on $P$, which defines the shortest path between $x^{*}$ and $y^{*}$.

The famous Hirsch conjecture (see [4]) states that for $m>n \geq 2$, diameter of $P$ is less than $m-n$. After 50 years of extensive research, this conjecture was disproved by Santos [9]. But the interest on the bound of the diameter of polytope is not reduced because this problem is not only hard but also has theoretical implication to the simplex method of the linear programming [2, 5, 6, 10. Recently, Bonifas et al. [1] derived an upper bound for a polytope with total unimodularity 1 i.e., for $A \in \mathbf{Z}^{m \times n}$, the upper bound is given as $\mathcal{O}\left(n^{3.5} \Delta^{2} \ln (n \Delta)\right)$, where $\Delta$ is the largest absolute value among all $(n-1) \times(n-1)$ sub-determinants of $A$. This short note shows that their method can

*US NRC, Office of Research, 11555 Rockville Pike, Rockville, 20850. Email: yaguang.yang@verizon.net.

${ }^{1}$ Although Bonifas et al. assumed that $A \in \mathbf{Z}^{m \times n}$, their results are applicable to more general settings as we will see in the derivations of this note. 
be extended to the case where $A \in \mathbb{R}^{m \times n}$. Moreover, if $A \in \mathbf{Z}^{m \times n}$, it also shows, by an example, that the new bound may be better than the bound of [1. We would also like to point out that parameters other than $\mathrm{m}$ and $\mathrm{n}$ (for example, smoothness parameters) in iteration bound for simplex method have been studied [3].

Without loss of generality, we may assume that the lengths of all row vectors of $A$ are one, which can easily be achieved by normalizing the row $A_{i}$, the $i$ th row of $A$, and dividing $b_{i}$ by $\left\|A_{i}\right\|$ for all $i$. This does not change the graph of the polytope $P$.

\section{Main results}

We follow the notations and definitions of Bonifas et al. [1]. First, assume that $P$ is non-degenerate, i.e., each vertex has exactly $n$ tight inequalities. Let $V$ be the set of all vertices of $P$. The normal cone $C_{v}$ of a vertex $v$ is the set of all vectors $c \in \mathbb{R}^{n}$ such that $v \in V$ is an optimal solution of the linear programming $\max \left\{c^{\mathrm{T}} x: x \in \mathbb{R}^{n}, A x \leq b\right\}$. Two vertices $u$ and $v$ are adjacent if and only if $C_{u}$ and $C_{v}$ share a facet. Let the unit ball

$$
B_{n}=\left\{x \in \mathbb{R}^{n}:\|x\|_{2} \leq 1\right\} .
$$

The volume of the union of the normal cones of $U \subseteq V$ is defined as

$$
\operatorname{vol}\left(S_{U}\right)=\operatorname{vol}\left(\cup_{v \in U} C_{v} \cap B_{n}\right),
$$

where $S_{v}=C_{v} \cap B_{n}$ is defined as the sphere cone of $C_{v}$.

For any two vertices $u$ and $v$ in $P$, starting from $u$ and $v$, the breadth-first-search finds all the neighbour vertices by iteration until a common vertex is discovered. The shortest path is no more than two times the number of iterations. Let $I_{j} \subseteq V$ be the set of vertices that have been discovered in $j$ th iteration. Clearly, if

$$
\operatorname{vol}\left(S_{I_{j}}\right) \geq \frac{1}{2} \operatorname{vol}\left(B_{n}\right)
$$

then, the common vertex must be found in less than $j$ iterations, i.e., the diameter is bounded by $2 j$. The rest effort is to estimate $j$ such that equation (1) holds.

The $(n-1)$-dimensional surface of a spherical cone $S$ that is not on the sphere is denoted as the dockable surface $D(S)$. Bonifas et al. showed the following:

Lemma 2.1 (Bonifas et al. [1]) Let $S$ be a (not necessarily convex) spherical cone with $\operatorname{vol}(S) \leq \frac{1}{2} \operatorname{vol}\left(B_{n}\right)$. Then,

$$
\frac{D(S)}{\operatorname{vol}(S)} \geq \sqrt{\frac{2 n}{\pi}}
$$

Let $\Delta$ denote the largest absolute value among all $(n-1) \times(n-1)$ sub-determinants of $A$ and $A_{v}$ be a $n \times n$ matrix of $A$ corresponding to a vertex $v \in V$, i.e., there is a $x$ satisfying $A x \leq b$ and $A_{v} x=b_{v}$ where $b_{v}$ is a sub-vector of $b$ whose index set is the 


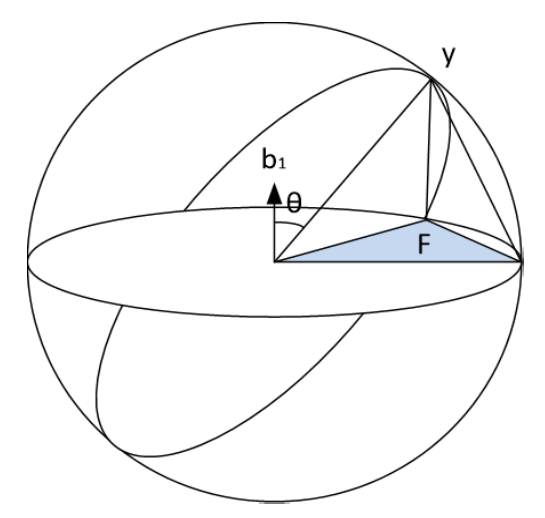

Figure 1: Proof of Lemma 2.2.

same as $A_{v}$. Denote $\operatorname{det}\left(A^{*}\right)=\min _{v \in V} \operatorname{det}\left(A_{v}\right)$, where $\operatorname{det}\left(A_{v}\right)$ is the volume of the box spanned by the (unit length) row vectors of $A_{v} \cdot \operatorname{det}\left(A^{*}\right)$ can be viewed as the condition number of polytope [12]. The next lemma is a modification of Lemma 3 of Bonifas et al. [1].

Lemma 2.2 Let $v$ be a vertex of $P$. Then, one has,

$$
\frac{D\left(S_{v}\right)}{\operatorname{vol}\left(S_{v}\right)} \leq \frac{n^{2.5} \Delta}{\operatorname{det}\left(A^{*}\right)}
$$

Proof: The proof uses the same idea of Bonifas et al. [1] for the case of $A \in \mathbb{R}^{m \times n}$. Let $F$ be a facet of a spherical cone $S_{v}$. Let $y$ be the vertex of $S_{v}$ not contained in the $(n-1)$ dimensional facet $F$. Let $Q$ be the convex hull of $F$ and $y$. We have $Q \subseteq S_{v}$ because $S_{v}$ is convex. Let $h_{F}$ be the Euclidean distance of $y$ from the hyperplane containing $F$, we have

$$
\operatorname{vol}\left(S_{v}\right) \geq \operatorname{vol}(Q)=\frac{\operatorname{area}(F) \cdot h_{F}}{n} .
$$

This yields

$$
\frac{D\left(S_{v}\right)}{\operatorname{vol}\left(S_{v}\right)}=\sum_{\text {facet } F} \frac{\operatorname{area}(F)}{\operatorname{vol}\left(S_{v}\right)} \leq n \sum_{\text {facet } F} \frac{1}{h_{F}} .
$$

Let $a_{1}, a_{2}, \ldots, a_{n}$ be the row vectors of $A_{v}$, and $b_{1}, b_{2}, \ldots, b_{n}$ be the column vectors of the adjugate of $A_{v}$. Clearly $A_{v}\left[b_{1}, b_{2}, \ldots, b_{n}\right]=\operatorname{det}\left(A_{v}\right) I$, where $I$ is an identity matrix. This means that $a_{1}^{\mathrm{T}} b_{1}=\operatorname{det}\left(A_{v}\right)$ and $b_{1} \perp\left\{a_{2}, \ldots, a_{n}\right\}$. Without loss of generality, assuming that $y$ lies on the ray generated by $a_{1}$ (actually $y=a_{1}$ because we assumed that the lengths of rows of $A$ are one), clearly, $h_{F}$ is length of the projection of $a_{1}$ onto $b_{1}$. Let $\theta$ be the angle described in Figure 1, noticing that the absolute value of each component of $b_{1}$ is less than or equal to $\Delta$, we have

$$
h_{F}=\left\|a_{1}\right\| \cos (\theta)=\left\|a_{1}\right\| \frac{a_{1}^{\mathrm{T}} b_{1}}{\left\|a_{1}\right\| \cdot\left\|b_{1}\right\|}=\frac{a_{1}^{\mathrm{T}} b_{1}}{\left\|b_{1}\right\|}=\frac{\operatorname{det}\left(A_{v}\right)}{\left\|b_{1}\right\|} \geq \frac{\operatorname{det}\left(A^{*}\right)}{\sqrt{n} \Delta} .
$$


Substituting this into (44) completes the proof.

The aforementioned two lemmas lead to the following claim.

Lemma 2.3 Let $P=\left\{x \in \mathbb{R}^{n}: A x \leq b\right\}$ be a general polytope with $A \in \mathbb{R}^{m \times n}$ and $m>n \geq 2$. Assume that all $(n-1) \times(n-1)$ sub-determinants of $A$ are bounded above by $\Delta$ and $\operatorname{det}\left(A_{v}\right)$ are bounded below by $\operatorname{det}\left(A^{*}\right)$. Let $I_{j} \subseteq V$ be a set of vertices with $\operatorname{vol}\left(I_{j}\right) \leq \frac{1}{2} \operatorname{vol}\left(B_{n}\right)$. Then the volume of the neighbourhood of $I_{j}$, denoted by $\operatorname{vol}\left(S_{N\left(I_{j}\right)}\right)$, satisfies

$$
\operatorname{vol}\left(S_{N\left(I_{j}\right)}\right) \geq \sqrt{\frac{2}{\pi}} \frac{\operatorname{det}\left(A^{*}\right)}{n^{2} \Delta} \cdot \operatorname{vol}\left(S_{I_{j}}\right) .
$$

Proof: Noticing that $D\left(S_{I_{j}}\right)$ is part of $\sum_{v \in N\left(I_{j}\right)} D\left(S_{v}\right)$ and using Lemma 2.1, we have

$$
\sum_{v \in N\left(I_{j}\right)} D\left(S_{v}\right) \geq D\left(S_{I_{j}}\right) \geq \sqrt{\frac{2 n}{\pi}} \cdot \operatorname{vol}\left(S_{I_{j}}\right) .
$$

Applying Lemma 2.2, we have

$$
\sum_{v \in N\left(I_{j}\right)} D\left(S_{v}\right) \leq \frac{n^{2.5} \Delta}{\operatorname{det}\left(A^{*}\right)} \sum_{v \in N\left(I_{j}\right)} \operatorname{vol}\left(S_{v}\right)=\frac{n^{2.5} \Delta}{\operatorname{det}\left(A^{*}\right)} \cdot \operatorname{vol}\left(S_{N\left(I_{j}\right)}\right) .
$$

Combining these two inequality gives

$$
\operatorname{vol}\left(S_{N\left(I_{j}\right)}\right) \geq \frac{\operatorname{det}\left(A^{*}\right)}{n^{2.5} \Delta} \sqrt{\frac{2 n}{\pi}} \cdot \operatorname{vol}\left(S_{I_{j}}\right)=\sqrt{\frac{2}{\pi}} \frac{\operatorname{det}\left(A^{*}\right)}{n^{2} \Delta} \cdot \operatorname{vol}\left(S_{I_{j}}\right)
$$

This completes the proof.

The main result of this short note follows from Lemma 2.3.

Theorem 2.1 Let $P=\left\{x \in \mathbb{R}^{n}: A x \leq b\right\}$ be a general polytope with $A \in \mathbb{R}^{m \times n}$ and $m>n \geq 2$. Assume that all $(n-1) \times(n-1)$ sub-determinants of $A$ are bounded above by $\Delta$ and $\operatorname{det}\left(A_{v}\right)$ are bounded below by $\operatorname{det}\left(A^{*}\right)$. Then, the diameter of the polytope $P$ is bounded by $\mathcal{O}\left(n^{3} \ln (n) \Delta\right)$ if $\operatorname{det}\left(A^{*}\right) \geq \frac{1}{2}$; for $\operatorname{det}\left(A^{*}\right)<\frac{1}{2}$, the bound is given by $j=\mathcal{O}\left(\frac{n^{3} \Delta}{\operatorname{det}\left(A^{*}\right)} \ln \left(\frac{n}{\operatorname{det}\left(A^{*}\right)}\right)\right)$.

Proof: We assume that the breadth-first-method starts from vertex $v$. For $j \geq 1$ and $\operatorname{vol}\left(S_{I_{j-1}}\right) \leq \frac{1}{2} \cdot \operatorname{vol}\left(B_{n}\right)$, using Lemma 2.3, we have

$$
\begin{aligned}
\operatorname{vol}\left(S_{I_{j}}\right) & \geq\left(1+\sqrt{\frac{2}{\pi}} \frac{\operatorname{det}\left(A^{*}\right)}{n^{2} \Delta}\right) \cdot \operatorname{vol}\left(S_{I_{j-1}}\right) \\
& \geq\left(1+\sqrt{\frac{2}{\pi}} \frac{\operatorname{det}\left(A^{*}\right)}{n^{2} \Delta}\right)^{j} \cdot \operatorname{vol}\left(S_{I_{0}}\right)
\end{aligned}
$$


where $S_{I_{0}}=S_{v}$ includes a simplex $J_{n}$ spanned by $n+1$ vertices composed of 0 and $n$ row vectors of $A_{v}$ (see Figure 11). Since the volume of $J_{n}$ is given by [8]

$$
\operatorname{vol}\left(J_{n}\right)=\frac{\operatorname{det}\left(A_{v}\right)}{n !} \geq \frac{\operatorname{det}\left(A^{*}\right)}{n !}
$$

we have

$$
\operatorname{vol}\left(S_{I_{0}}\right) \geq \operatorname{vol}\left(J_{n}\right) \geq \frac{\operatorname{det}\left(A^{*}\right)}{n !} .
$$

Assuming $n$ is even (which is easy to derive the result but the order of the estimation remains the same for odd $n$ ), we have

$$
\operatorname{vol}\left(B_{n}\right)=\frac{\pi^{\frac{n}{2}}}{\left(\frac{n}{2}\right) !}
$$

The condition $\operatorname{vol}\left(S_{I_{j}}\right) \leq \frac{1}{2} \cdot \operatorname{vol}\left(B_{n}\right)$ implies

$$
\frac{1}{2} \cdot \operatorname{vol}\left(B_{n}\right)=\frac{1}{2} \frac{\pi^{\frac{n}{2}}}{\left(\frac{n}{2}\right) !} \geq \operatorname{vol}\left(S_{I_{j}}\right) \geq\left(1+\sqrt{\frac{2}{\pi}} \frac{\operatorname{det}\left(A^{*}\right)}{n^{2} \Delta}\right)^{j} \frac{\operatorname{det}\left(A^{*}\right)}{n !}
$$

or

$$
\pi^{\frac{n}{2}} \geq 2 \frac{\left(\frac{n}{2}\right) !}{n !} \operatorname{det}\left(A^{*}\right)\left(1+\sqrt{\frac{2}{\pi}} \frac{\operatorname{det}\left(A^{*}\right)}{n^{2} \Delta}\right)^{j}
$$

For $0 \leq c \leq 1$, it has $\ln (1+c) \geq c / 2$. Therefore, we can rewrite (12) as

$$
\begin{aligned}
\frac{n}{2} \ln \pi & \geq \ln \left(2 \frac{\left(\frac{n}{2}\right) !}{n !} \operatorname{det}\left(A^{*}\right)\right)+j \ln \left(1+\sqrt{\frac{2}{\pi}} \frac{\operatorname{det}\left(A^{*}\right)}{n^{2} \Delta}\right) \\
& \geq \ln \left(\frac{1}{n^{n / 2}}\right)+\ln \left(2 \operatorname{det}\left(A^{*}\right)\right)+j \sqrt{\frac{1}{2 \pi}} \frac{\operatorname{det}\left(A^{*}\right)}{n^{2} \Delta} .
\end{aligned}
$$

Therefore, we have

$$
\frac{n}{2} \ln (n \pi) \geq \ln \left(2 \operatorname{det}\left(A^{*}\right)\right)+j \sqrt{\frac{1}{2 \pi}} \frac{\operatorname{det}\left(A^{*}\right)}{n^{2} \Delta} .
$$

This shows $j=\mathcal{O}\left(\frac{n^{3} \Delta \ln (n \pi)}{\operatorname{det}\left(A^{*}\right)}\right)$ if $\operatorname{det}\left(A^{*}\right) \geq \frac{1}{2}$. For $\operatorname{det}\left(A^{*}\right)<\frac{1}{2}$,

$$
\begin{aligned}
& \frac{n}{2} \ln (n \pi) \geq \frac{n}{2} \ln \left(2 \operatorname{det}\left(A^{*}\right)\right)+j \sqrt{\frac{1}{2 \pi}} \frac{\operatorname{det}\left(A^{*}\right)}{n^{2} \Delta} \\
\Longrightarrow & \frac{n}{2} \ln \left(\frac{n \pi}{2 \operatorname{det}\left(A^{*}\right)}\right) \geq j \sqrt{\frac{1}{2 \pi}} \frac{\operatorname{det}\left(A^{*}\right)}{n^{2} \Delta},
\end{aligned}
$$

this shows $j=\mathcal{O}\left(\frac{n^{3} \Delta}{\operatorname{det}\left(A^{*}\right)} \ln \left(\frac{n}{\operatorname{det}\left(A^{*}\right)}\right)\right)$. 
Remark 2.1 The upper bound in Theorem 2.1 is not only related to $n$, like the ones of [7, 10, 11], but also to the condition numbers of the vertices of $A_{v}$. If the rays of all $S_{v}$ are almost perpendicular, then $\operatorname{det}\left(A^{*}\right)$ will be close to one. Otherwise, if for some $v$, the rays of $S_{v}$ are almost linear dependent, then $\operatorname{det}\left(A^{*}\right)$ will be close to zero, and the diameter bound of the polytope given in Theorem 2.1 will increase significantly. Therefore, $\operatorname{det}\left(A^{*}\right)$ can be viewed as the condition number of the polytope.

We conclude this short note by examining a high dimensional cubic polytope.

\section{Example 2.1}

$$
\left[\begin{array}{cccccc}
1 & 0 & 0 & \ldots & 0 & 0 \\
0 & 1 & 0 & \ldots & 0 & 0 \\
0 & 0 & 1 & \ldots & 0 & 0 \\
\vdots & \vdots & \vdots & \ddots & 0 & 0 \\
0 & 0 & 0 & \ldots & 1 & 0 \\
0 & 0 & 0 & \ldots & 0 & 1 \\
-1 & 0 & 0 & \ldots & 0 & 0 \\
0 & -1 & 0 & \ldots & 0 & 0 \\
0 & 0 & -1 & \ldots & 0 & 0 \\
\vdots & \vdots & \vdots & \ddots & 0 & 0 \\
0 & 0 & 0 & \ldots & -1 & 0 \\
0 & 0 & 0 & \ldots & 0 & -1
\end{array}\right]\left[\begin{array}{c}
x_{1} \\
x_{2} \\
\vdots \\
\vdots \\
x_{m-1} \\
x_{m}
\end{array}\right] \leq\left[\begin{array}{c}
1 \\
1 \\
\vdots \\
\vdots \\
1 \\
1 \\
0 \\
0 \\
\vdots \\
\vdots \\
0 \\
0
\end{array}\right]
$$

Clearly, all rows are normalized and the absolute value of any sub-determinant of $A$ is either 0 or 1 . Applying Theorem 10 of [1] gives an upper bound of $\mathcal{O}\left(n^{3.5} \ln (n)\right)$; applying Theorem 2.1 gives an upper bound of $\mathcal{O}\left(n^{3} \ln (n)\right)$. Therefore, for this problem, the new bound proposed in this note is better than the one in Theorem 10 of [1]. However, this example does not imply that the derived bound is better than Bonifas et al's in general.

\section{3 acknowledgment}

This author thanks the anonymous reviewers for their valuable comments.

\section{Declarations of interest:}

This research did not receive any specific grant from funding agencies in the public, commercial, or not-for-profit sectors. 


\section{References}

[1] N. Bonifas, M. Di Summa, F. Eisenbrand, N. Hähnle, and M. Niemeier, On subdeterminants and the diameter of polyhedra, Discrete Comput. Geom, 52 (2014) 102-115.

[2] S. Borgwardt, J. A. De Loera and E. Finhold, The diameters of network-flow polytopes satisfy the Hirsch conjecture, Mathematical Programming 171 (2018) 283-309.

[3] , D. Dadush and S. Huiberts, A friendly smoothed analysis of the simplex method, (2017) https://arxiv.org/abs/1711.05667

[4] G.B. Dantzig, Linear programming and extension, Princeton University Press, New Jersey, 1963.

[5] A. Del Pia and C. Michini, On the Diameter of Lattice Polytopes, Discrete and Computational Geometry, 55 (2016) 681-687.

[6] A. Deza and L. Pournin, Improved bounds on the diameter of lattice polytopes, Acta Mathematica Hungarica, 154 (2018) 457-469.

[7] G. Kalai, D.J. Kleitman, A quasi-polynomial bound for thediameter of graphs of polyhedra, Bull. Am. Math. Soc., 26 (1992) 315-216.

[8] P. Stein, A note on the volume of a simplex, The American Mathematical Monthly, 73 (1966) 299-301.

[9] F. Santos, A countexample to the Hirsch conjecture, Annals of Math., 176 (2012) 383-412.

[10] N. Sukegawa, Improving bounds on the diameter of a polyhedron in high dimensions, Discrete Mathematics, 340 (2017) 2134-2142.

[11] M. J. Todd, An improved Kalai-Kleitman bound for the diameter of a polyhedron, SIAM Journal on Discrete Mathematics, 26 (2014) 1944-1947.

[12] Y. Yang, A new condition number of the eigenvalue and its application in control theory, Journal of Computational Mathematics, 7 (1989) 15-22. 